\title{
H INFINITY ATTITUDE CONTROLLER DESIGN FOR A RIGID-FLEXIBLE SATELLITE CONSIDERING THE PARAMETRIC UNCERTAINTY
}

ALAIN GIACOBINI DE SOUZA LUIZ CARLOS GADELHA DE SOUZA

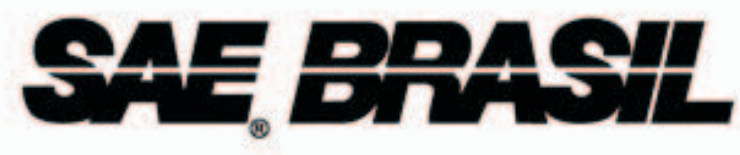

AFFILIATED TO

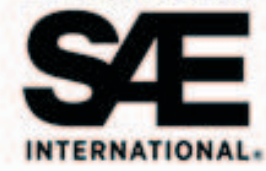

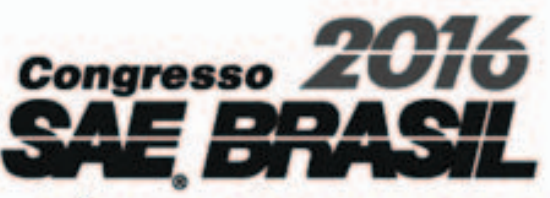

$25^{\text {th }}$ SAE BRASIL International

Congress and Display São Paulo, Brasil October, $25^{\text {th }}$ to $27^{\text {th }}$ 


\title{
H Infinity Attitude Controller Design for a Rigid-Flexible Satellite Considering the Parametric Uncertainty
}

\author{
Alain Giacobini Souza1, Luiz Carlos Gadelha Souza ${ }^{2}$ \\ ${ }^{1,2}$ National Institute for Space Research (INPE) \\ ${ }^{2}$ Brasília University (UnB)
}

Copyright (C) 2016 SAE International

\begin{abstract}
In designing of the Attitude Control System (ACS) is important take into account the influence of the structure's flexibility, since they can interact with the satellite rigid motion, mainly, during translational and/or rotational maneuver, damaging the ACS pointing accuracy. In the linearization and reduction of the rigid-flexible satellite mathematic model, usually one loses some important information associated with the satellite true dynamical behavior. One way to recovery this information is include to the ACS design parametric and not parametric uncertainties of the system. The $\mathrm{H}$ infinity control method is able to take into account the parametric uncertainty in the control law design, so the controller becomes more robust. This paper presents the design of a robust controller using the $\mathrm{H}$ infinity control technique to control the attitude of a rigid-flexible satellite. The satellite model is represented by a flexible beam connected to a central rigid hub considering a set of parametric uncertainties. The results of the simulations have shown that the control law designed with the H-infinity control method was robust enough as to support the action of uncertainty and control the rigid flexible satellite attitude and suppressing vibrations.
\end{abstract}

\section{Introduction}

Satellites are design with specific functions and for accomplish them; the system need be able to execute some maneuvers around his center of mass (attitude maneuvers). These functions are: turning the solar panels facing the sun, stabilize the satellite after the orbital acquisition, point the cameras and/or sensors to the Earth or other planet, moon and star [1]. For conducting this kind of maneuver is necessary have a lot of sensors and actuators with a good accuracy and precision. With that, for managerial all that, one need design a control system called attitude control system (ACS).

Appendages flexibles plays an important role in satellites system, this system can be: powers supplies, communication, weather services, radiators and a variety of space researches [2]. Thus the dynamic of these flexible structures have to be included in the controller design. Then for the attitude control design it is important to studies the effects of the vibrational motion of the flexibles structures [3], because this effects can interact with the satellite rigid motion, mainly, during translational and/or rotational maneuver, damaging the ACS pointing accuracy $[1,2]$.

Modeling the vibrational dynamics isn't a simple task [4]. Exist many methods to write the mathematic model of this candy of system, but all this methods are not precisely because this dynamics have a Page 1 of 7 nonlinear comportment, then for trade with this problem one makes some assumptions and linearize the equations of the dynamics [5]. The cost of the linearization process is: lose some important information associated with the satellite true dynamical behavior. One way to recovery this information is to include in the ACS design parametric and non-parametric uncertainties of the system [6].

The $\mathrm{H}$ infinity control method has the property to incorporate the uncertainties in its formulation, resulting in a control law more robust [5] able to suppress the uncertainties and this control law it has the potential to control the real system with all his nonlinearities [6].

In nowadays due to tight integration and the increasing complexity of the systems that there is a synergy in various fields such as the use of aerospace control techniques in non-space systems. Than this technique will be present can be useful for other non-space systems here exist an interaction between the rigid and flexibles dynamics [7]. Examples of these non-space structures are: robotic arms, unmanned aerial vehicles (UAV's) [8] and others. This technic explores the proprieties of robustness of the $\mathrm{H}$ infinity control method, given a solution to the problem of the tradeoff between the rigid and flexible dynamics in attitude maneuvers.

This paper designs one ACS using the $\mathrm{H}$ infinity method considering a parametric uncertainty in a satellite. The model used is similar to the China Brazil Earth Resources Satellite - CBERS, as shown in Figure 1.

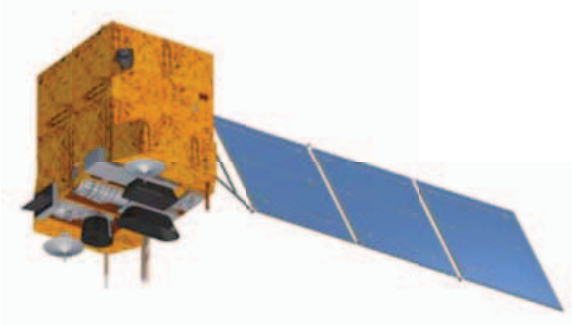

Figure 1. China Brazil Earth Resources Satellite - CBERS. Source: (http://www.cbers.inpe.br/sobre satelite/descricao cbers3e4.php) Accessed on: $02-01-2016$

\section{Dynamic Model With Parametric Uncertain}

The rigid-flexible satellite was modeled like a flexible rotatory beam $[3,5]$. The actuator is located in the central part, and it is responsible 
for the control of the horizontal planar movement of the rigid structure and by the elastic displacement, where $\left(X_{0}, Y_{0}\right)$ and $(\mathrm{X}, \mathrm{Y})$ are the coordinates system before and after deformation, respectively. The rigid central hub has radius $\mathrm{R}$, the actuator rotor has viscous friction $b_{m}$, and inertia $J_{\text {RотоR }}$ and develops a torque $\tau(\mathrm{t})$; the flexible beam has uniform linear mass density $\rho$, uniform bending stiffness EI and length L; the flexible beam deformation is $\mathrm{w}(\mathrm{x}, \mathrm{t})$; and the tip mass is $m_{\text {tip }}$ and inertia $J_{\text {tip }} ; \alpha(\mathrm{t})$ represent the tip angle and $\theta(\mathrm{t})$ is the rigid angle displacement; and $\mathrm{s}(\mathrm{x})$ represent the distance between the referential axis to an element of mass in the link.

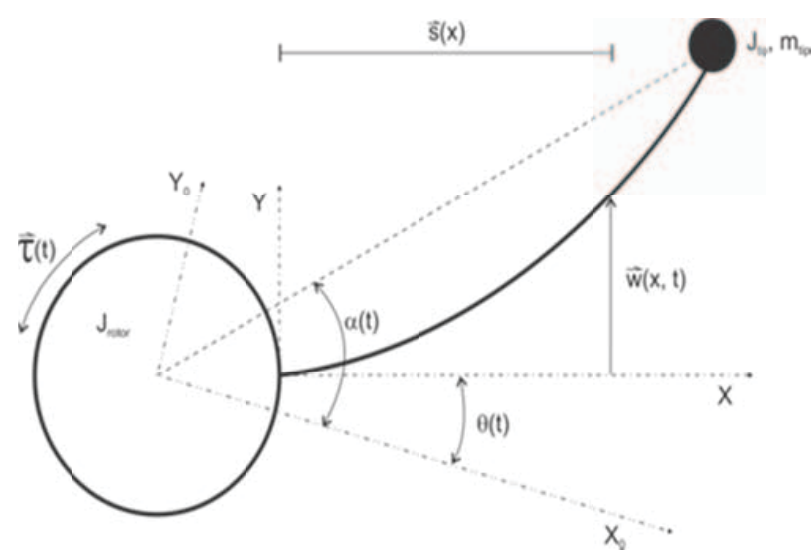

Figure 2. Satellite Analogous Representation

The flexible link is considered an Euler-Bernoulli beam. The equations of motion are derived using the Lagrangian approach combined with the assumed modes method [3], admitting two vibrations mode.

In [3] was demonstrate that the Lagragian for this model is given by:

$$
\begin{gathered}
\mathcal{L}=\frac{1}{2} \dot{\theta}\left[J_{\text {rotor }}+\mathbf{q}^{T} \rho \int_{0}^{L} \boldsymbol{\phi} \boldsymbol{\phi}^{T} d x \mathbf{q}+\rho \frac{1}{3}(R+L)^{3}\right. \\
\left.+m_{\text {tip }}\left(\mathbf{q} \boldsymbol{\phi}_{L} \boldsymbol{\phi}_{L}^{T} \mathbf{q}+(R+L)^{2}\right)+J_{t i p}\right] \\
+\frac{1}{2} \dot{\theta}\left[2 \rho \dot{\mathbf{q}}^{T} \int_{0}^{L} \boldsymbol{\phi}(R+s) d x+2 m_{t i p} \dot{\mathbf{q}}^{T} \boldsymbol{\phi}_{L}(R+L)+J_{t i p} \dot{\mathbf{q}}^{T} \boldsymbol{\phi}^{\prime}\right] \\
+\frac{1}{2}\left[\rho \dot{\mathbf{q}}^{T} \int_{0}^{L} \boldsymbol{\phi} \boldsymbol{\phi}^{T} d x \dot{\mathbf{q}}+m_{t i p} \dot{\mathbf{q}}^{T}\left(\boldsymbol{\phi}_{L} \boldsymbol{\phi}_{L}^{T}\right) \dot{q}+J_{t i p} \dot{\mathbf{q}}\left(\boldsymbol{\phi}^{\prime} \boldsymbol{\phi}^{\prime T}\right) \dot{\mathbf{q}}\right] \\
-\frac{1}{2} \mathbf{q}^{T} E I \int_{0}^{L} \boldsymbol{\phi} \boldsymbol{\phi}^{T} d x \mathbf{q}
\end{gathered}
$$

Where $\theta$ is the rigid angular displacement, $\mathbf{q}$ the deflection vector and $\phi$ is the function of the modal form.

Applying the Lagragian methodology $[3,5]$, one was the have the following nonlinear dynamic, represented by a matrix form:

$\left[\begin{array}{cc}\mathbf{I}_{t}+\mathbf{q}^{T} \mathbf{C}_{r r} \mathbf{q} & \mathbf{M}_{r f}^{T} \\ \mathbf{M}_{r f} & \mathbf{M}_{f f}\end{array}\right]\left[\begin{array}{c}\theta \\ \ddot{\mathbf{q}}\end{array}\right]+\left[\begin{array}{cc}b_{m} & \mathbf{0} \\ \mathbf{0} & \boldsymbol{B}_{\mathrm{ff}}\end{array}\right]\left[\begin{array}{c}\dot{\theta} \\ \dot{\mathbf{q}}\end{array}\right]+\left[\begin{array}{cc}\mathbf{0} & \mathbf{0} \\ \mathbf{0} & \boldsymbol{K}_{\mathrm{ff}}\end{array}\right]\left[\begin{array}{l}\theta \\ \mathbf{q}\end{array}\right]=\left[\begin{array}{l}\tau \\ \mathbf{0}\end{array}\right]$ with,

Page 2 of 7

$$
\begin{aligned}
& \mathbf{B}_{f f}=k_{e} E I \int_{0}^{L} \boldsymbol{\phi}^{\prime \prime} \boldsymbol{\phi}^{\prime \prime T} d x \\
& \mathbf{K}_{f f}=E I \int_{0}^{L} \boldsymbol{\phi}^{\prime \prime} \boldsymbol{\phi}^{\prime \prime T} d x \\
& \mathbf{K}_{f f}=E I \int_{0}^{L} \boldsymbol{\phi}^{\prime \prime} \boldsymbol{\phi}^{\prime \prime T} d x \\
& \mathbf{M}_{f f}=\rho \int_{0}^{L} \boldsymbol{\phi} \boldsymbol{\phi}^{T} d x+m_{t i p} \boldsymbol{\phi}_{L} \boldsymbol{\phi}_{L}^{T}+\frac{1}{2} J_{t i p} \boldsymbol{\phi}_{L}^{\prime} \boldsymbol{\phi}_{L}^{\prime T}
\end{aligned}
$$

To obtain a linear model the nonlinear terms $\mathbf{q}^{\mathrm{T}} \mathbf{C}_{\mathrm{rr}} \mathbf{q}, \dot{\theta} \mathbf{q}^{\mathrm{T}} \mathbf{C}_{\mathrm{rr}} \mathbf{q}$ and $-\dot{\theta}^{2} \mathbf{C}_{r r} \mathbf{q}$ are neglected due to the fact that they are relatively very small [6]. So writing eq. (2) in standard form $\mathbf{M} \ddot{x}+\mathbf{D} \dot{x}+\mathbf{K} x=\mathbf{Q} u$ (M is the mass matrix, $\mathbf{D}$ is the damping matrix and $\mathbf{K}$ is the rigid matrix), one has the dynamics linearized model, in space state form, given by

$$
\left[\begin{array}{c}
\dot{\mathbf{X}}_{1} \\
\dot{\mathbf{X}}_{2}
\end{array}\right]=\left[\begin{array}{cc}
0 & \mathbf{I} \\
-\mathbf{M}^{-1} \mathbf{K} & -\mathbf{M}^{-1} \mathbf{D}
\end{array}\right]\left[\begin{array}{l}
\mathbf{X}_{1} \\
\mathbf{X}_{2}
\end{array}\right]+\left[\begin{array}{c}
0 \\
\mathbf{M}^{-1} \mathbf{Q}
\end{array}\right] \mathbf{u}
$$

with $\mathbf{X}_{1}=\theta$ and $\mathbf{X}_{2}=\mathbf{q}=\left[\begin{array}{ll}q_{1} & q_{2}\end{array}\right]$ is the state's vectors and the control $\mathbf{u}=-\mathbf{K}_{H_{\infty}} \boldsymbol{X}$, where $\mathbf{K}_{H_{\infty}}$ is the gain calculate by the $\mathrm{H}$ infinity method [3].

For imprecisely known parameter values of $\mathbf{M}$, $\mathbf{D}$ e $\mathbf{K}$ the dynamic behavior [6] can be described in a general form like:

$$
\mathbf{M}=\overline{\mathbf{M}}\left(\mathbf{I}+p_{m} \boldsymbol{\delta}_{m}\right), \mathbf{D}=\overline{\mathbf{D}}\left(\mathbf{I}+p_{d} \boldsymbol{\delta}_{d}\right) \text { and } \mathbf{K}=\overline{\mathbf{K}}\left(\mathbf{I}+p_{k} \boldsymbol{\delta}_{k}\right)
$$

where the terms $\overline{\mathbf{M}}, \overline{\mathbf{D}}$ and $\overline{\mathbf{K}}$ are the nominal values, $p_{m}, p_{d}$ and $p_{k}$ are uncertain values and $\boldsymbol{\delta}_{m}, \boldsymbol{\delta}_{d}$ and $\boldsymbol{\delta}_{k}$ are the amplitude of the uncertain $\left(-1 \leq \delta_{\mathrm{m}, \mathrm{d}, \mathrm{k}} \leq 1\right)$. For this study case, $p_{m, d, k}=30 \%$.

\section{H Infinity Control Method with Uncertainty}

One great advantage of the $\mathrm{H}$ infinity control method is the possibility of designing robust controllers with respect to structured uncertainty at the same time that is possible to obtain good performance with respect to unstructured uncertainty $[6,9,10]$.

The $\mathrm{H}$ infinity control method consist basically calculate a gain $\mathbf{K}$ that minimizing the $\mathrm{H}$ infinity norm of the closed loop transfer function,

$$
\begin{gathered}
|| F_{l}(\mathbf{P}, \mathbf{K}) \|_{\infty}=\max _{\omega} \bar{\sigma}\left(F_{l}(\mathbf{P}, \mathbf{K})(j \omega)\right) \\
|| F_{l}(\mathbf{P}, \mathbf{K}) \|_{\infty}<\gamma
\end{gathered}
$$

where $F_{l}(\mathbf{P}, \mathbf{K})$ is the linear fractional transformation (LFT) of $\mathbf{P}$ and $\mathbf{K}$

$$
F_{l}(\mathbf{P}, \mathbf{K}) \omega=\mathbf{P}_{11}+\mathbf{P}_{12} \mathbf{K}\left(\mathbf{I}-\mathbf{P}_{22} \mathbf{K}\right)^{-1} \mathbf{P}_{21}
$$

The $\gamma$ factor is obtain numerically from a state-space realization as the smallest value of $\gamma$ such that the Hamiltonian matrix $\mathbf{H}$ has no eigenvalues on the imaginary axis $[3,6,10]$,

$$
\mathbf{H}=\left[\begin{array}{cc}
\mathbf{A}+\mathbf{B R}^{-1} \mathbf{D}^{T} \mathbf{C} & \mathbf{B R}^{-1} \mathbf{B}^{T} \\
-\mathbf{C}^{T}\left(\mathbf{I}+\mathbf{D R}^{-1} \mathbf{D}^{T}\right) \mathbf{C} & -\left(\mathbf{A}+\mathbf{B R}^{-1} \mathbf{D}^{T} \mathbf{C}\right)^{T}
\end{array}\right]
$$


where $\mathbf{A}, \mathbf{B}, \mathbf{C}$ and $\mathbf{D}$ are the space state matrices and $\mathbf{R}=\gamma^{2} \mathbf{I}-\mathbf{D}^{T} \mathbf{D}$. We can also include weight functions in the system like the Figure 3

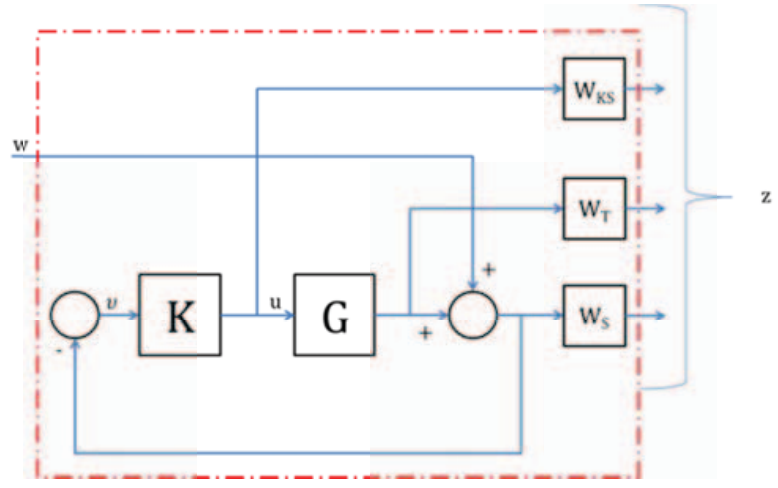

Figure 3. Weight functions

This weight function assume the form [8]:

$$
W_{s}=\frac{\frac{s}{M}+\omega_{b}}{s+\omega_{b} A}, W_{T}=\frac{s+\frac{\omega_{b c}}{M}}{A s+\omega_{b c}}, W_{K S}=c t e
$$

The parameters of the weight functions: $A$ is correlated with the steady state, $\omega_{b}$ is the desired bandwidth of the sensitivity function, $M$ is correlated with the overshoot, $\omega_{b c}$ is the desired bandwidth of the complementary sensitivity function [9].

And the generalized plant $\mathbf{P}$ is given by:

$$
\mathbf{P}=\left[\begin{array}{cc}
0 & W_{K S} \\
0 & W_{T} \\
W_{S} \mathbf{I} & W_{S} \mathbf{G} \\
-\mathbf{I} & -\mathbf{G}
\end{array}\right]
$$

The parameter $\mathbf{G}$ is the plant model. The schema of the generic plant with uncertain is given by the Figure 4 .

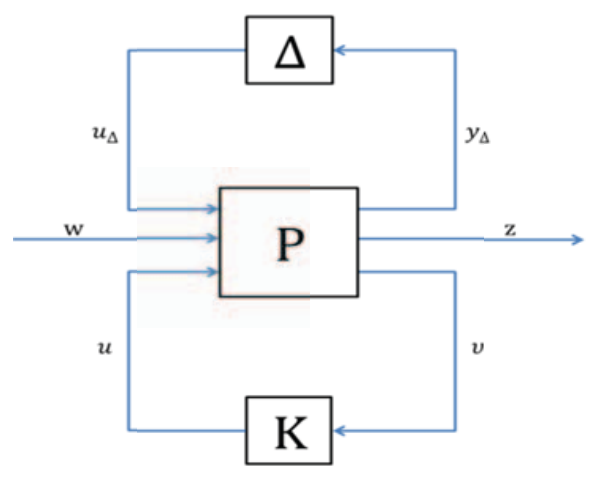

Figure 4. Standard configuration

In Figure 4 it is possible to observe the interaction between the generic plant (P), controller $(\mathbf{K})$ and uncertain $(\boldsymbol{\Delta})$.

The conditions of robust stabilization are obtain $\mathbf{K}$ then obeys the follow relations:

$$
|| \mathbf{\Delta K}(\mathbf{I}+\mathbf{G K})^{-1}||_{\infty}<1
$$

and the optimization problem is given by the functional [10]:

$$
\min _{\mathbf{K} \text { stabilising }}|| \mathbf{\Delta K}(\mathbf{I}+\mathbf{G K})^{-1} \|_{\infty}
$$

\section{Controller Design}

For the controller one considers the system of equations linearized like show in eq. (4) In real system the matrices $\mathbf{M}, \mathbf{D}$ e $\mathbf{K}$ cannot be exactly determinate, with that we consider a uncertain in this values. Then, we can re-write the Equation (4) in the follow form [3, 11]:

$$
\overline{\mathbf{M}}\left(\mathbf{I}+p_{m} \boldsymbol{\delta}_{m}\right) \ddot{x}+\overline{\mathbf{D}}\left(\mathbf{I}+p_{d} \boldsymbol{\delta}_{d}\right) \dot{x}+\overline{\mathbf{K}}\left(\mathbf{I}+p_{k} \boldsymbol{\delta}_{k}\right) x=\tau
$$

The matrix $\overline{\mathbf{M}}^{-1}$ can be represented by a linear fraction transformation (LFT) in $\delta_{m}$, like:

$$
\overline{\mathbf{M}}^{-1}=\overline{\mathbf{M}}^{-1}\left(\mathbf{I}+p_{m} \boldsymbol{\delta}_{m}\right)^{-1}=F_{U}\left(M_{m i}, \delta_{m}\right)
$$

in other form:

$$
M_{m i}=\left[\begin{array}{ll}
-\mathbf{I} p_{m} & \overline{\mathbf{M}}^{-1} \\
-\mathbf{I} p_{m} & \overline{\mathbf{M}}^{-1}
\end{array}\right]
$$

In an analog way the parameters $D=\overline{\mathbf{D}}\left(\mathbf{I}+p_{d} \boldsymbol{\delta}_{d}\right)$ and $K=\overline{\mathbf{K}}(\mathbf{I}+$ $p_{k} \boldsymbol{\delta}_{\mathrm{k}}$ ) can be express in a LTF (Linear Transfers Function) form:

$$
M_{d}=\left[\begin{array}{cc}
0 & \overline{\mathbf{D}} \\
\mathbf{I} p_{d} & \overline{\mathbf{D}}
\end{array}\right], M_{k}=\left[\begin{array}{cc}
0 & \overline{\mathbf{K}} \\
\mathbf{I} p_{k} & \overline{\mathbf{K}}
\end{array}\right]
$$

For show the interconnections between the uncertainties, we write a blog diagram where the inputs are $\left(\delta_{m}, \delta_{d}\right.$ and $\left.\delta_{k}\right)$ and the output are $\left(v_{m}, v_{d}\right.$ and $\left.v_{k}\right)$. See Figure 4.

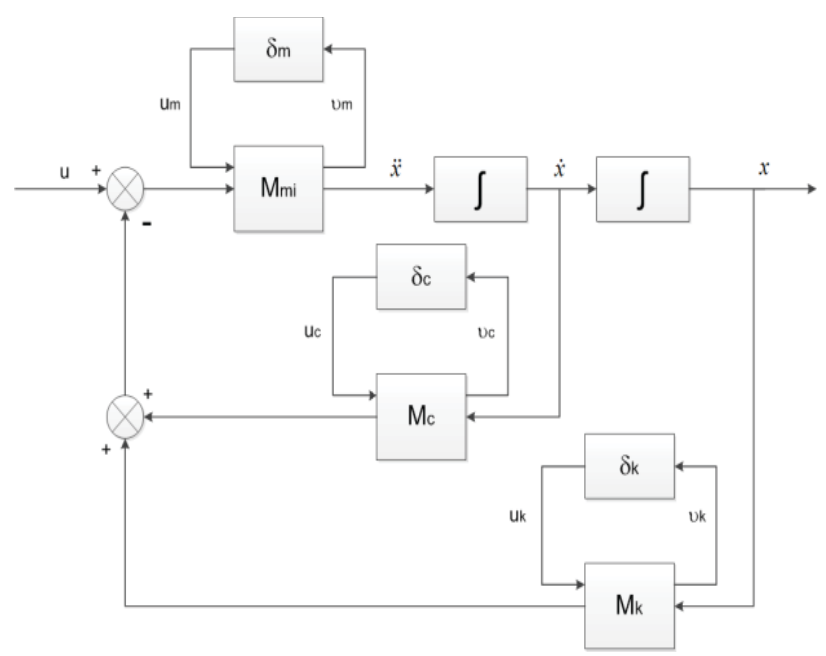

Figure 5. Block diagram of the system with uncertainties.

In mathematical words one has:

Page 3 of 7 


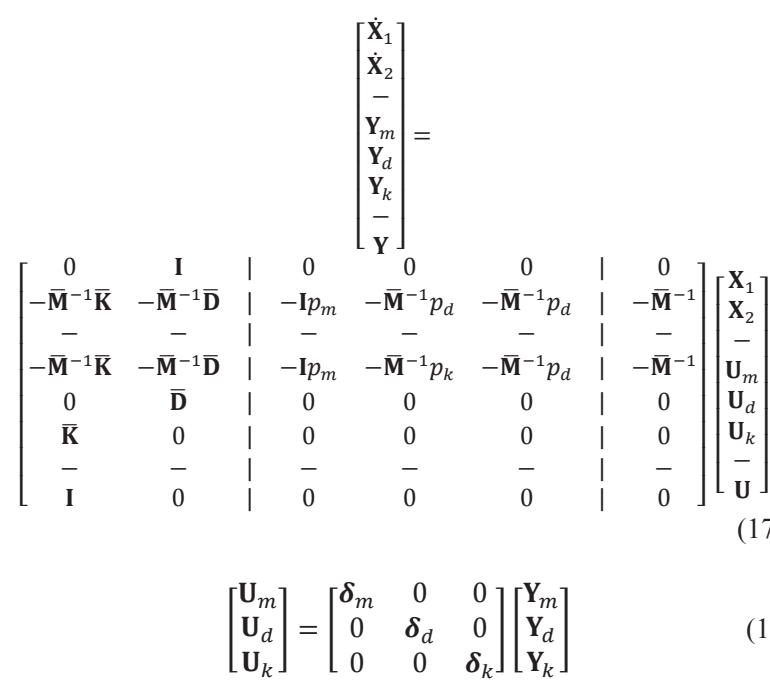

Let the $G_{m d s}$ denotes the input/output of the system, which take into account the uncertainty parameters as show in the Figure 6.

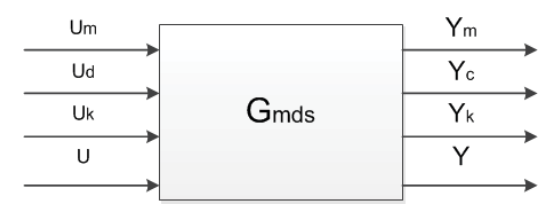

Figure 6. Input/Output diagram.

In the Figure $6 G_{m d s}$ one has the input vector $\left(\mathbf{U}_{m}, \mathbf{U}_{d}, \mathbf{U}_{k}, \mathbf{U}\right)$, the output vector $\left(\mathbf{Y}_{m}, \mathbf{Y}_{k}, \mathbf{Y}_{d}, \mathbf{Y}\right)$ and two space vector $\left(\mathbf{X}_{1}, \mathbf{X}_{2}\right)$.

The uncertain in this problem actuate in the matrices $\mathbf{M}, \mathbf{D}$ and $\mathbf{K}$. Than the uncertain amplitude is given by $\boldsymbol{\delta}_{m, k, d}$. These matrices are diagonal, with order 3 and their value range is:

$$
\delta_{m, k, d}=\left[\begin{array}{ccc}
\delta_{11} & 0 & 0 \\
0 & \delta_{22} & 0 \\
0 & 0 & \delta_{33}
\end{array}\right], \delta_{i, j}=\left\{\begin{array}{l}
-1 \leq \delta_{11} \leq 1 \\
-1 \leq \delta_{22} \leq 1 \\
-1 \leq \delta_{33} \leq 1
\end{array}\right.
$$

With that ones have 27 possibilities of $\boldsymbol{\delta}_{m, d, k}$, or else, ones have 27 cases of uncertainties for each matrix $\mathbf{M}, \mathbf{D}$ and $\mathbf{K}$. For this work is considered the same $\boldsymbol{\delta}$ for the three matrices $\boldsymbol{\delta}=\boldsymbol{\delta}_{m}=\boldsymbol{\delta}_{d}=\boldsymbol{\delta}_{k}$.

\section{Simulations}

The objective of the control simulation is stabilize the tip angle $(\alpha)$ of the flexible link in a neutral position $\left(0^{\circ}\right)$. The angle $\alpha$ is given by:

$$
\alpha(t)=\arctan \left(\frac{w(x, t)}{L+R}\right)+\theta
$$

When the tip comes to the neutral positions is important to see if the the vibration was suppress, because with that we can assure that the control law design was robust enough to absorb the uncertainties.

Appling the $\mathrm{H}$ infinity control method in the plant model eq 17 , one obtains the fallow results for the initial response (the link is in the position $\theta=1^{0}$ ) for the 27 possible cases of uncertainty. An

Page 4 of 7 important observation is the controller gain chances for every case of uncertain, is to say the $\mathrm{H}_{\infty}$ control method researches the most robust gain as possible. All simulations are made for a time interval of $180 \mathrm{~s}$.

For the simulation the constant parameter are: $\mathrm{R}=0.05 \mathrm{~m}, \mathrm{~b}_{\mathrm{m}}=0.15$ $\mathrm{m}^{2} / \mathrm{s}, \quad \mathrm{J}_{\text {rotor }}=0.3 \mathrm{kgm}^{2}, \quad \mathrm{~L}=1 \mathrm{~m}, \quad \rho=2700 \quad \mathrm{~kg} / \mathrm{m}, \quad \mathrm{ke}=0.03$, $\mathrm{EI}=18.4 \mathrm{Nm}^{2}, \mathrm{~m}_{\text {tip }}=0.25 \mathrm{~kg}$ and $\mathrm{J}_{\text {tip }}=0.04 \mathrm{kgm}^{2}$. One obtains:

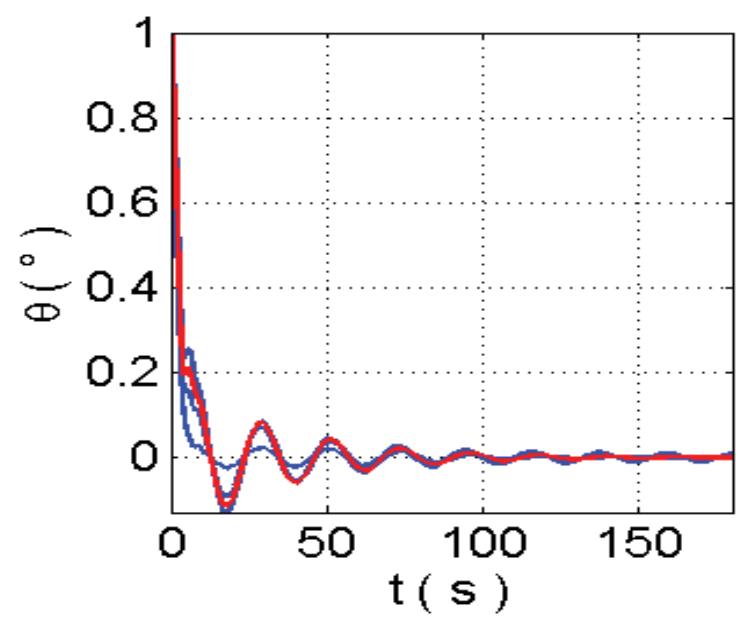

Figure 7. Variation of the attitude angle $\theta$, the red line is the nominal case and the blue lines are the uncertainty cases

In the Figure 7 ones have all 27 cases for the attitude angle $\theta$. The red line is the nominal case. The systems return to the equilibrium position approximately in 180 s for 24 cases. Is clear to see that some cases the answer is better than the nominal case, showing the influence of some parameters are more expressive than others in the sense of minor overshoot and time rise.

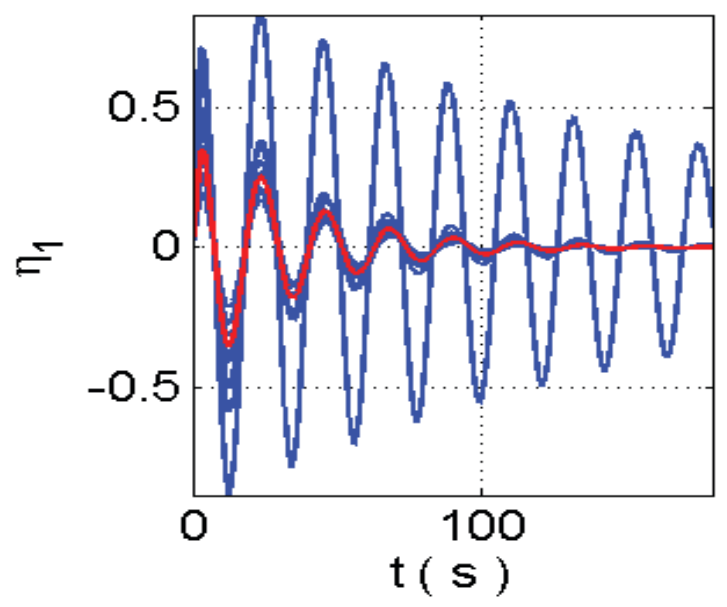

Figure 8. Variation of the first flexible mode $\boldsymbol{q}_{\mathbf{1}}$, the red line is the nominal case and the blue lines are the uncertainty cases

In the Figure 8 ones have all 27 cases for the first flexible mode, generalized coordinate $q_{1}$. The red line is the nominal case. For 14 cases the first mode archive the equilibrium position in the $180 \mathrm{~s}$. 


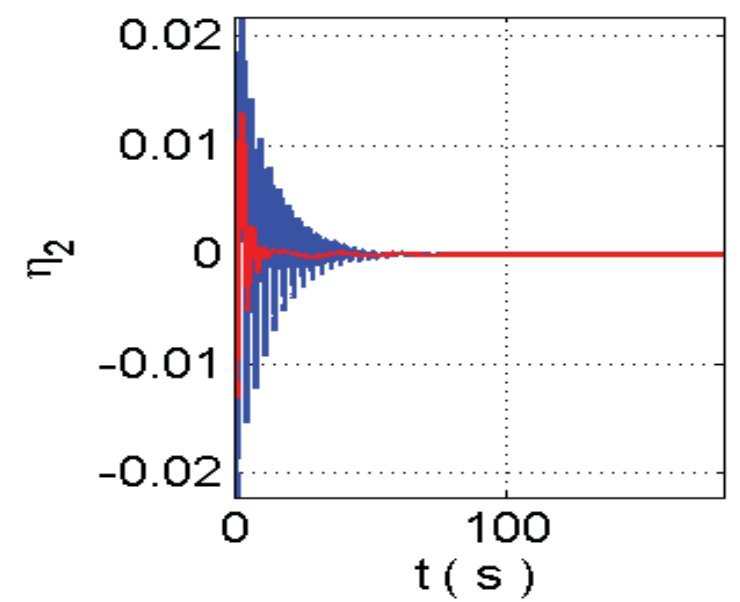

Figure 9. Variation of the second flexible mode $\boldsymbol{q}_{\mathbf{2}}$, the red line is the nominal case and the blue lines are the uncertainty cases

In the Figure 9 ones have all 27 cases for the second flexible mode, generalized coordinate $q_{2}$. The red line is the nominal case. In this case the second mode, go to the equilibrium, for all cases, in approximately $100 \mathrm{~s}$.

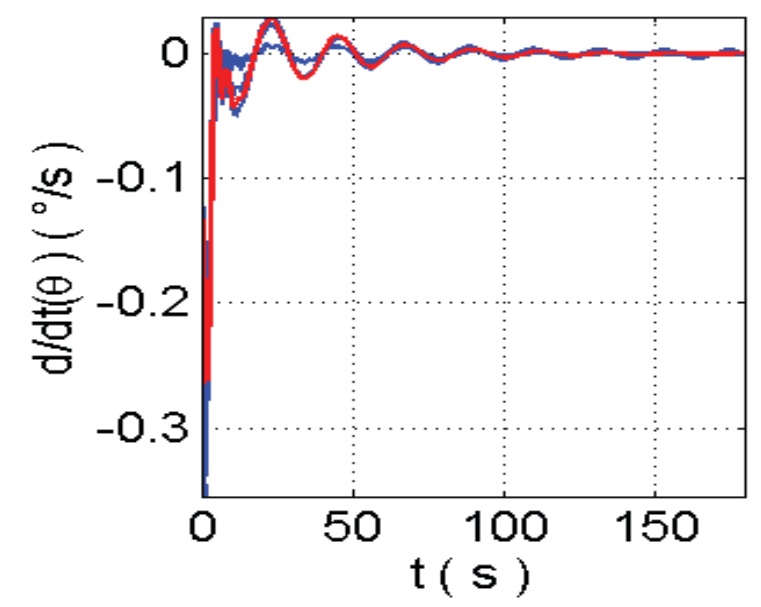

Figure 10 . Variation of the attitude velocity $\dot{\boldsymbol{\theta}}$, the red line is the nominal case and the blue lines are the uncertainty cases

In the Figure 10 ones have all 27 cases for the attitude velocity $\dot{\theta}$. The red line is the nominal case. For 24 cases the attitude velocity return to $0 \% \mathrm{~s}$ in approximately $180 \mathrm{~s}$.

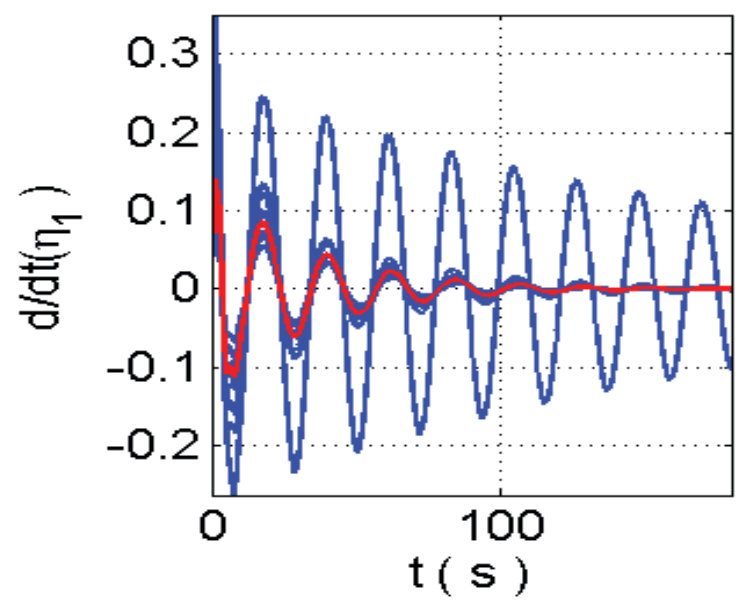

Figure 11. Variation rate of the firts flexible mode $\boldsymbol{q}_{\mathbf{1}}$, the red line is the nominal case and the blue lines are the uncertainty cases

In the Figure 11 ones have all 27 cases for the variation of the first flexible mode, generalized coordinate $\dot{q}_{1}$. The red line is the nominal case. For 14 cases the first mode archive the equilibrium position in the 180 s.

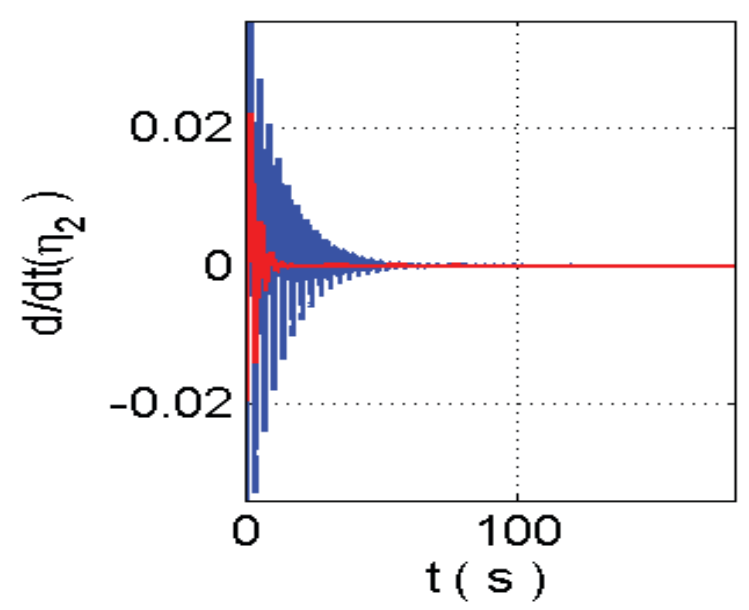

Figure 12. Variation rate of the second flexible mode $\dot{q}_{2}$, the red line is the nominal case and the blue lines are the uncertainty cases

In the Figure 12 ones have all 27 cases for the variation of the second flexible mode, generalized coordinate $\dot{q}_{2}$. The red line is the nominal case. In this case the second mode, go to the equilibrium, for all cases, in approximately $100 \mathrm{~s}$. 


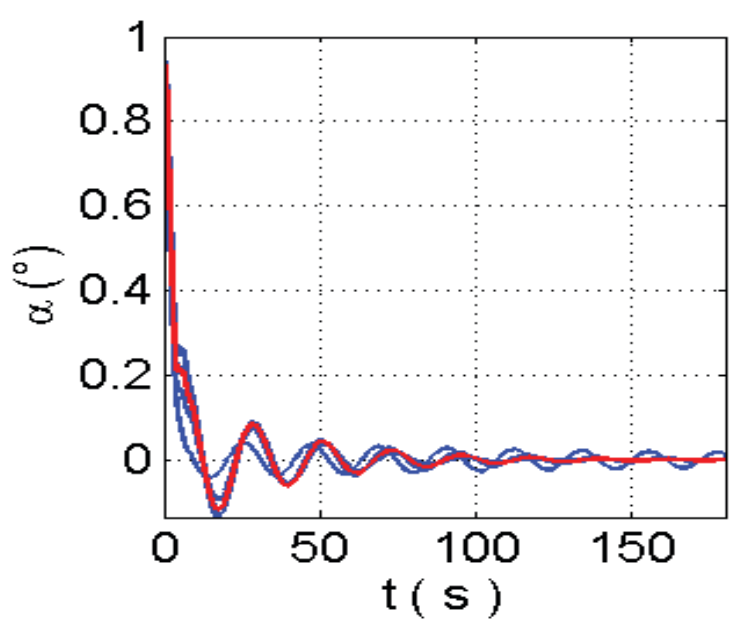

Figure 13. Angular response of the flexible angle $\alpha$, the red line is the nominal case and the blue lines are the uncertainty cases

In the Figure 13 ones have all 27 cases for the tip mass angle $\alpha$. The red line is the nominal case. Like we can see in the Figure 1 the angle $\alpha$ represent de angular position of the tip mass locate in the extremity of the beam. The response of this angle shows that the tip mass return to the equilibrium position in 24 cases in 180s approximately.

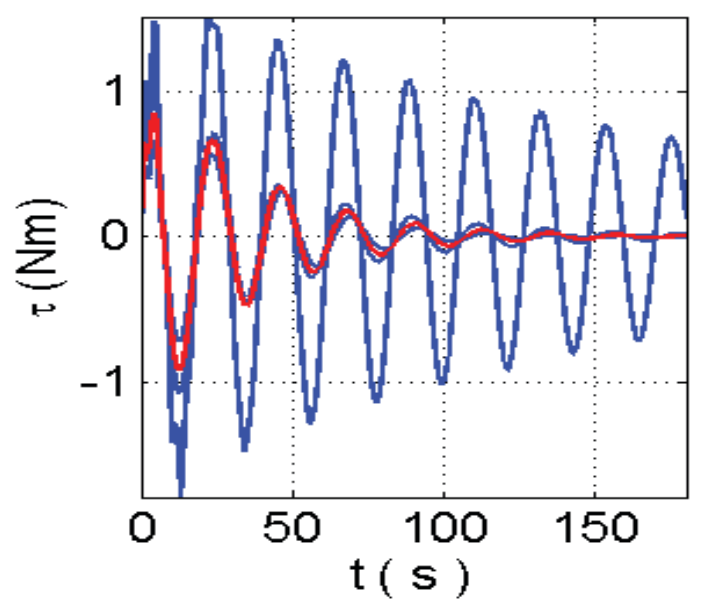

Figure 14. Response of the control effort $\tau$, the red line is the nominal case and the blue lines are the uncertainty cases

In the Figure 14 ones have all 27 cases for the control signal, torque $\tau$. The red line is the nominal case. The response of the action of the torque shows that for some 15 cases the system archive the equilibrium in 180 s.

\section{Conclusion}

This paper presents an attitude controller design for a rigid-flexible satellite using the $\mathrm{H}$ infinity method considering a parametrical uncertainty, using model and simulation it was possible to analyses the interaction between the rigid and flexible dynamics in an attitude maneuver and studies the robust proprieties of the $\mathrm{H}$ infinity when a set of parametric uncertainties is insert in the system.
The results of the simulations shows that the control law design, for the rigid flexible satellite, was able to support the parametric uncertainty making the system return to an equilibrium position in approximately 180 s for $56 \%$ cases and for the other cases the rise time was biggest, but the control method was robust enough to suppress the uncertainty and suppress the vibrations. In other words, the control law design was able to calculate new gains that provided a good response a sense of don't destabilize the system.

For the futures works, we think to explore every single case, with objective to find which set of uncertainty that has greater influence on the plant and using other plants from the UAV and/or robotic domain.

\section{References}

1. Sidi, M. J. "Spacecraft dynamics and control: A pratical engineering approach". NewYork, NY: Cambridge University Press, 1997.

2. Junkins, J. L, Kim, Y. "Introduction to dynamics and control of flexible structures". Washington, DC: American Institute of Aeronautics and Astronautics (AIAA), 1993.

3. Souza, A.G, Souza, L. C. G, "H infinity controller design to a rigid-flexible satellite with two vibration modes," Journal of Physics: Conference Series, vol. 641, pp. 012030, 2015.

4. Souza, L.C.G, Souza, A.G. "Satellite Attitude Control System Design considering the Fuel Slosh Dynamics," Shock and Vibration, vol. 2014, Article ID 260206, 8 pages, 2014. doi: $10.1155 / 2014 / 260206$

5. Bigot, P. G.; Souza, L. C. G. "Design of non-linear controller for a flexible rotatory beam using state-dependent riccati equation (sdre) control". Proceedings of the 2013 International Conference on Systems, Control and Informatics, p. 288 - 295, 2013.

6. Pinheiro, E.R., Souza L.C.G, "Design of the Microsatellite Attitude Control System Using the Mixed $\mathrm{H} 2 / \mathrm{H} \infty$ Method via LMI Optimization," Mathematical Problems in Engineering, vol. 2013, Article ID 257193, 8 pages, 2013.

7. Pinheiro, E.R., Souza L.C.G, "Design of the Microsatellite Attitude Control System Using the Mixed H2/Ho Method via LMI Optimization," Mathematical Problems in Engineering, vol. 2013, Article ID 257193, 8 pages, 2013. doi:10.1155/2013/25719.

8. P. Gasbarri, R. Monti, C. De Angelis, M. Sabatini, "Effects of uncertainties and flexible dynamic contributions on the control of a spacecraft full-coupled model", Acta Astronautica, Volume 94, Issue 1, January-February 2014, Pages 515-526, ISSN 00945765, http://dx.doi.org/10.1016/j.actaastro.2012.08.018.

9. H. C. Ferreira, R. S. Baptista, J. Y. Ishihara and G. A. Borges, "Disturbance rejection in a fixed wing UAV using nonlinear $\mathrm{H} \infty$ state feedback," 2011 9th IEEE International Conference on Control and Automation (ICCA), Santiago, 2011, pp. 386-391.

10. Zhou, K, Doyle, J. C, "Essentials of robust control". Upper Saddle River, NJ:Prentice-Hall, 1998.

11. Skogestad, S, Postlethwaite, I. "Multivariable feedback control: analysis and design". Chichester, UK: John Wiley and Sons, 2001.

12. D.W., P. P. G.; M.M., K. Robust control design: with matlab. London, UK: Springer, 2005

\section{Contact Information}

Alain Giacobini de Souza Post Graduate Student 
National Institute for Space Research-INPE

Course of Space Engineering and Technology/Option Space

Mechanics and Control-ETE/CMC

Av. dos Astronautas, 1758 - Satellite Building

São José dos Campos, SP, 12227-010, Brazil

Phone: (12) 32087422 / 6191, E-mail: alaingiacobini@gmail.com

Luiz Carlos Gadelha de Souza

Professor and Senior Researcher

Brasília University (UnB)

Campus Gama

Área Especial de Indústria-A-UnB,

Brasília, DF, 72444-240, Brazil

Phone: (61) 31078901 / 8904 / 8907, E-mail: lcgadelha@gmail.com

\section{Acknowledgments}

The authors would like to thank CAPES and INPE/DMC.

\section{Definitions/Abbreviations}

ACS

Attitude Control System

CBERS

China Brazil Earth

Resources Satellite

UAV

Unmanned Aerial Vehicles

All rights reserved. No part of this publication may be reproduced, stored in a retrieval system, or transmitted, in any form or by any means, electronic, mechanical, photocopying, recording, or otherwise, without the prior written permission of SAE.

ISSN 0148-7191

Copyright (c) 2016 SAE International.

Positions and opinions advanced in this paper are those of the author(s) and not necessarily those of SAE. The authors solely responsible for the content of the paper.

Page 7 of 7 